\title{
SEQUENCES IN THE RANGE OF A VECTOR MEASURE WITH BOUNDED VARIATION
}

\author{
CÁNDIDO PIÑEIRO
}

(Communicated by Palle E. T. Jorgensen)

\begin{abstract}
Let $X$ be a Banach space. We consider sequences $\left(x_{n}\right)$ in $X$ lying in the range of a measure valued in a superspace of $X$ and having bounded variation. Among other results, we prove that G.T. spaces are the only Banach spaces in which those sequences are actually in the range of an $X^{* *}$-valued measure of bounded variation.
\end{abstract}

In [PR] it is proved that a Banach space $X$ is finite-dimensional if and only if every null sequence $\left(x_{n}\right)$ (equivalently, every compact set) in $X$ lies inside the range of an $X$-valued measure of bounded variation. In view of this result, a natural question arises: Which Banach spaces have the property that every null sequence lies inside the range of a vector measure of bounded variation, if we do not mind the measure is not $X$-valued? We start by explaining some basic notation used in this paper. In general, our operator and vector measure terminology and notation follow [Ps] and [DU]. We only consider real Banach spaces. If $X$ is a such space, the phrase "range of an $X$-valued measure" always means a set of the form $\operatorname{rg}(F)=\{F(E): E \in \Sigma\}$, where $\Sigma$ is a $\sigma$-algebra of subsets of a set $\Omega$ and $F: \Sigma \rightarrow X$ is countably additive.

Definition 1. Let $X$ be a Banach space. We say that a subset $A$ of $X$ lies in the range of a vector measure of bounded variation (we shorten to a vector bvmeasure) provided there exist a Banach space $X_{0}$, an isometry $J: X \rightarrow X_{0}$ and a vector measure $F: \Sigma \rightarrow X_{0}$ with bounded variation so that $J(A) \subset \operatorname{rg}(F)$.

We are mainly interested in the case $A$ is countable. In the lemma below we collect some elementary facts.

Lemma 2. Let $X$ be a Banach space. If $\left(x_{n}\right)$ is a bounded sequence in $X$, we consider the linear operator $T:\left(\alpha_{n}\right) \in l_{1} \rightarrow \sum \alpha_{n} x_{n} \in X$.

Then the following assertions hold:

(i) $\left(x_{n}\right)$ is in the range of an $X$-valued bv-measure iff $T$ is Pietsch-integral.

(ii) $\left(x_{n}\right)$ lies inside the range of a vector bv-measure iff $T$ is 1-summing.

(iii) $\left(x_{n}\right)$ is in the range of an $X^{* *}$-valued bv-measure iff $T$ is integral.

Received by the editors March 23, 1994.

1991 Mathematics Subject Classification. Primary 46G10; Secondary 47B10.

This research was supported by the D.G.I.C.Y.T., PB 90-893.

(c) 1995 American Mathematical Society 
Proof. (i) Although it is well known, we include its proof. Let $F: \Sigma \rightarrow X$ a measure of bounded variation $\mu$ such that $\left\{x_{n}: n \in \mathbb{N}\right\} \subset \operatorname{rg}(F)$. Choose $A_{n} \in$ $\Sigma$ so that $F\left(A_{n}\right)=x_{n}$ for all $n \in \mathbb{N}$, and define two operators $P: l_{1} \rightarrow L^{\infty}(\mu)$ and $Q: L^{\infty}(\mu) \rightarrow X$ by

$$
P\left(\alpha_{n}\right)=\sum \alpha_{n} \chi_{A_{n}} \text { and } Q(f)=\int f d F .
$$

Since $T=Q P$, it suffices to prove that $Q$ is Pietsch-integral. By [P1] $Q$ is 1 -summing. Then $Q$ is Pietsch-integral because $L^{\infty}(\mu)$ is isometric to a $\mathscr{C}(K)$ space [DU]. Conversely, if $T$ is Pietsch-integral, then $T$ admits a factorization

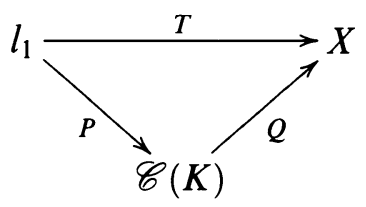

where $P$ is continuous and $Q$ is 1 -summing [DU]. By [PR, Proposition 1.3] there exists an $X$-valued measure $F$ of bounded variation such that $\overline{Q\left(B_{\mathscr{C}(K)}\right)}=$ $\operatorname{rg}(F)$. Hence $\left\{x_{n}: n \in \mathbb{N}\right\} \subset \operatorname{rg}(\|P\| F)$.

(ii) Suppose $T$ is 1 -summing. If $J$ denotes the natural isometry into $l_{\infty}\left(B_{X^{*}}\right)$, then $J T$ is Pietsch-integral. By (i) $\left(J x_{n}\right)$ lies inside the range of an $l_{\infty}\left(B_{X^{*}}\right)$-valued bv-measure. On the other hand, if $J: X \rightarrow X_{o}$ is an isometry and $F: \Sigma \rightarrow X_{o}$ is a bv-measure so that $\left\{J x_{n}: n \in \mathbb{N}\right\} \subseteq \operatorname{rg}(F)$, then (i) tells us that $J T$ is Pietsch-integral and, therefore, 1-summing. So is $T$ since $J$ is an isometry.

(iii) It is obvious since integral and Pietsch-integral operators into $X^{* *}$ are the same [DU].

Remark 3. Recall that a sequence $\left(x_{n}\right)$ in $X$ is said to belong to $l_{w}^{2}(X)$ provided we have $\sum\left|\left\langle x_{n}, x^{*}\right\rangle\right|^{2}<+\infty$ for any $x^{*} \in X^{*}$. If $T:\left(\alpha_{n}\right) \in l_{1} \rightarrow$ $\sum \alpha_{n} x_{n} \in X$ is 1 -summing, then $T$ admits a factorization

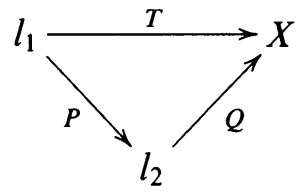

where $P$ and $Q$ are continuous with $\|P\| \leq 1$. Hence, there is a sequence $\left(z_{n}\right) \in l_{w}^{2}(X)$ so that

$$
Q\left(\alpha_{n}\right)=\sum \alpha_{n} z_{n} \quad \text { for any }\left(\alpha_{n}\right) \in l_{2} .
$$

Consequently, the class of sequences lying in the range of a bv-measure and the class of sequences which are contained in a weakly 2 -summable segment $\left\{\sum \alpha_{n} z_{n}:\left(\alpha_{n}\right) \in l_{2},\left\|\left(\alpha_{n}\right)\right\|_{2} \leq 1\right\}$ are equal.

A particular class of those sequences are the sequences belonging to $l_{w}^{2}(X)$. In [AD] it is proved that such a sequence $\left(x_{n}\right)$ lies inside the range of the $X$-valued measure $F$ defined by

$$
F(A)=2 \sum_{n}\left(\int_{A} r_{n}(t) d t\right) x_{n}
$$


for any Lebesgue measurable subset $A$ of $[0,1]$. But, in general this measure is not of bounded variation. Nevertheless, if $X$ has type 2 and $\left(x_{n}\right) \in l_{a}^{2}(X)$ (the Banach space of all sequences $\left(x_{n}\right)$ such that $\left.\left\|\left(x_{n}\right)\right\|_{2}=\left(\sum\left\|x_{n}\right\|^{2}\right)^{1 / 2}<+\infty\right)$, then $F$ has bounded variation. To see this, let $\left\{A_{1}, \ldots, A_{p}\right\}$ be a finite family of pairwise disjoint Lebesgue measurable subsets of $[0,1]$. Then

$$
\begin{aligned}
\sum_{i=1}^{p}\left\|\sum_{k=1}^{n}\left(\int_{A_{i}} r_{k}(t) d t\right) x_{k}\right\| & =\sum_{i=1}^{p}\left\|\int_{A_{i}}\left(\sum_{k=1}^{n} r_{k}(t) x_{k} d t\right)\right\| \\
\leq \int_{0}^{1}\left\|\sum_{k=1}^{n} r_{k}(t) x_{k}\right\| d t & \leq\left(\int_{0}^{1}\left\|\sum_{k=1}^{n} r_{k}(t) x_{k}\right\|^{2} d t\right)^{1 / 2} \leq T_{2}(X)\left\|\left(x_{n}\right)\right\|_{2}
\end{aligned}
$$

where $T_{2}(X)$ is the type 2 constant of $X$. Finally, letting $n \rightarrow \infty$ we obtain

$$
\sum_{i=1}^{p}\left\|F\left(A_{i}\right)\right\| \leq 2 T_{2}(X)\left\|\left(x_{n}\right)\right\|_{2} \text {. }
$$

So, $F$ has bounded variation.

If every unconditionally summable sequence $\left(x_{n}\right)$ in $X$ satisfies $\sum\left\|x_{n}\right\|^{2}<$ $+\infty$, we say that $X$ has Orlicz property. It is known that cotype 2 spaces have Orlicz property [Ps]. Hence, if $X$ has type $2, X^{*}$ has Orlicz property because the dual space $X^{*}$ has cotype 2 whenever $X$ has type 2 . In view of these results, it seems interesting to characterize the Banach spaces $X$ in which every sequence $\left(x_{n}\right) \in l_{a}^{2}(X)$ is in the range of an $X$-valued bv-measure. Next Theorem proves that those spaces are the Banach spaces whose dual have Orlicz property.

Theorem 4. Let $X$ be a Banach space. The following statements are equivalent:

(i) Every sequence $\left(x_{n}\right) \in l_{a}^{2}(X)$ is in the range of an $X$-valued bv-measure.

(ii) Every sequence $\left(x_{n}\right) \in l_{a}^{2}(X)$ is in the range of an $X^{* *}$-valued $b v$ measure.

(iii) $X^{*}$ has Orlicz property.

Proof. (i) $\Rightarrow$ (ii) is obvious and (iii) $\Rightarrow(\mathrm{i})$ is straightforward.

(ii) $\Rightarrow$ (iii) The natural inclusion $I: l_{a}^{2}(X) \rightarrow I\left(l_{1}, X\right)$ is linear and, having closed graph, continuous. Since $\left(x_{n}\right)=\lim _{n \rightarrow \infty}\left(x_{1}, \ldots, x_{n}, 0,0, \ldots\right)$ for any $\left(x_{n}\right) \in l_{a}^{2}(X)$, it follows that $I$ takes $l_{a}^{2}(X)$ into $N\left(l_{1}, X\right)$ (note that $N\left(l_{1}, X\right.$ ) is a subspace of $I\left(l_{1}, X\right)$ because $\left(l_{1}\right)^{*}$ has the metric approximation property). By transposition we obtain that $B\left(X, l_{1}\right)$ is taken into $l_{a}^{2}\left(X^{*}\right)$. So $X^{*}$ has Orlicz property.

Now we are going to answer our first question.

Theorem 5. Let $X$ be a Banach space. The following statements are equivalent:

(i) The unit ball of $X$ lies in the range of a vector $b v$-measure.

(ii) Every bounded sequence in $X$ is in the range of a vector bv-measure.

(iii) Every null sequence in $X$ is in the range of a vector $b v$-measure.

(iv) $X$ is isomorphic to a Hilbert space.

Proof. Obviously (i) $\Rightarrow$ (ii) $\Rightarrow$ (iii) .

(iii) $\Rightarrow$ (iv) Again we consider the natural isometry $J: X \rightarrow l_{\infty}\left(B_{X^{*}}\right)$. By hypothesis, $J$ maps null sequences of $X$ into sequences lying inside the range 
of an $l_{\infty}\left(B_{X^{*}}\right)$-valued bv-measure. From [Pñ] it follows that $J^{*}$ is 1 -summing. In particular, $J^{*}$ is 2-summing, so it admits a factorization

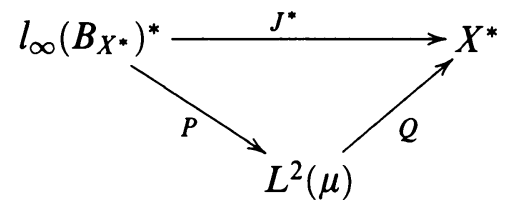

where $P$ and $Q$ are continuous linear maps. As $J^{*}$ is a surjection, so is $Q: L^{2}(\mu) \rightarrow X^{*}$. This proves that $X^{*}$ is isomorphic to a Hilbert space.

(iv) $\Rightarrow$ (i) If $X$ is isomorphic to a Hilbert space, then the map

$$
A:\left(\xi_{x}\right) \in l_{1}\left(B_{X}\right) \rightarrow \sum \xi_{x} x \in X
$$

is 1-summing. Hence, $J A$ is Pietsch-integral. In a similar way to the proof of Lemma 1(i), we can prove that $J\left(B_{X}\right)$ lies inside the range of an $l_{\infty}\left(B_{X^{*}}\right)$ valued bv-measure.

Next, we shall prove that every sequence in $X$, lying in the range of a vector bv-measure, is actually in the range of an $X^{* *}$-valued bv-measure if and only if $X^{*}$ is a G.T. space. Following [Ps] we will say that a Banach space $X$ satisfies Grothendieck's Theorem (in short G.T.) if

$$
B\left(X, l_{2}\right)=\Pi_{1}\left(X, l_{2}\right) .
$$

The next theorem shows that G.T. spaces are the only spaces in which every sequence lying in the range of a vector bv-measure actually lies inside the range of an $X^{* *}$-valued bv-measure.

Theorem 6. Let $X$ be a Banach space. The following are equivalent:

(i) $X^{*}$ is a G.T. space.

(ii) $\Pi_{1}\left(l_{1}, X\right)=I\left(l_{1}, X\right)$.

Proof. (i) $\Rightarrow$ (ii) Let $T: l_{1} \rightarrow X$ be 1-summing. Then $T$ admits a factorization:

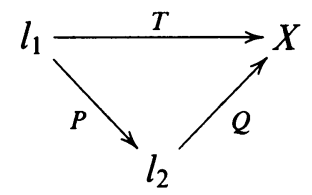

So $T^{*}=Q^{*} P^{*}$. By hypothesis, $Q^{*}: X^{*} \rightarrow l_{2}$ is 1 -summing. Hence, $T^{*}: X^{*} \rightarrow$ $l_{\infty}$ is integral.

(ii) $\Rightarrow$ (i) Recall that 1-summing and 2-summing operators on $l_{1}$ are the same because $l_{1}$ has cotype $2[\mathrm{Ps}$ ]. Consequently, by the open mapping theorem there is a positive constant $c$ such that

$$
i(T) \leq c \pi_{2}(T) \text { for any } T \in \Pi_{2}\left(l_{1}, X\right) .
$$

Then, we have

$$
i(T) \leq c \pi_{2}(T) \text { for any } n \text { and any } T \in \Pi_{2}\left(l_{1}^{n}, X\right) .
$$

But the integral norm and the nuclear norm of an operator defined on a finitedimensional space are equal. So, we can write (1) in the form

$$
\nu(T) \leq c \pi_{2}(T) \text { for any } n \text { and for any } T: l_{1}^{n} \rightarrow X .
$$


Now, by the duality argument [T, p. 18], $\left(1^{\prime}\right)$ yields

$$
\pi_{2}(T) \leq c\|T\| \text { for any } n \text { and for any } T: X \rightarrow l_{1}^{n} .
$$

The result follows from [Ps, Theorem 6.2].

\section{FINAL NOTES AND EXAMPLES}

1. It is well known that the unit ball of $l_{2}$ is the range of and $l_{2}$-valued countably additive measure [AD]. Therefore, so is every weakly 2-summable segment $\left\{\sum \alpha_{n} z_{n}:\left\|\left(\alpha_{n}\right)\right\|_{2} \leq 1\right\}$. Then Remark 3 shows that every sequence lying in the range of a vector bv-measure is actually in the range of an $X$-valued countably additive measure. Nevertheless, the converse result is not true. To see this, take the space $l_{\infty}$. As mentioned earlier, there are null sequences in $l_{\infty}$ which do not lie in the range of an $l_{\infty}$-valued bv-measure but they do in the range of an $l_{\infty}$-valued measure since $\left(l_{\infty}\right)^{*}$ is an $L^{1}(\mu)$-space [PR].

2. We know from Lemma 2 that a sequence $\left(x_{n}\right)$ in $X$ lies in the range of an $X^{* *}$-valued bv-measure iff $T:\left(\alpha_{n}\right) \in l_{1} \rightarrow \sum \alpha_{n} x_{n} \in X$ is integral, equivalently, iff $T^{*}: x^{*} \in X^{*} \rightarrow\left(\left\langle x_{n}, x^{*}\right\rangle\right)_{n} \in l_{\infty}$ is 1 -summing. We have obtained the following characterization of these sequences:

A sequence $\left(x_{n}\right)$ in $X$ lies in the range of an $X^{* *}$-valued bv-measure iff there is a positive constant $c$ such that

$$
\sum_{n}\left|\left\langle x_{n}, \phi(n)\right\rangle\right| \leq c \sup \left\{\sum_{n}\left|\left\langle\phi(n), x^{* *}\right\rangle\right|:\left\|x^{* *}\right\| \leq 1\right\}
$$

for any $\phi: \mathbb{N} \rightarrow X^{*}$ so that the set $\{n \in \mathbb{N}: \phi(n) \neq 0\}$ is finite.

Necessity is obvious. To prove that $T^{*}$ is 1 -summing if (2) holds, we apply Ky Fan's Lemma [P2] to the collection $\mathscr{F}$ of all functions $\Phi$ defined by

$$
\Phi(\mu)=\sum_{n}\left(\left|\left\langle x_{n}, \phi(n)\right\rangle\right|-c \int_{B_{X^{* *}}}\left|\left\langle\phi(n), x^{* *}\right\rangle\right| d \mu\right),
$$

where $\phi: \mathbb{N} \rightarrow X^{*}$ is as before and $\mu$ is a probability measure on the unit ball of $X^{* *}$ endowed with the topology $\sigma\left(X^{* *}, X^{*}\right) /_{B_{X^{* *}}}$. If $W\left(B_{X^{* *}}\right)$ denotes the set of all such probability measures, then every $\Phi \in \mathscr{F}$ is convex and continuous on $W\left(B_{X^{* *}}\right)$ endowed with the $\mathrm{w}^{*}$-topology. Using Dirac measures, we can prove that, given $\Phi \in \mathscr{F}$, there is $\mu \in W\left(B_{\left.X^{* *}\right)}\right.$ so that $\Phi(\mu) \leq 0$. Since $\mathscr{F}$ is concave, it follows that there is $\mu \in W\left(B_{X} \cdot\right)^{\circ}$ satisfying $\Phi(\mu) \leq 0$ for any $\Phi \in \mathscr{F}$. In particular, it follows that

$$
\left|\left\langle x_{n}, x^{*}\right\rangle\right| \leq c \int_{B_{X^{* *}}}\left|\left\langle x^{*}, x^{* *}\right\rangle\right| d \mu
$$

for any $n \in \mathbb{N}$ and any $x^{*} \in X^{*}$. This proves that $T^{*}$ is 1-summing.

3. Integral and Pietsch-integral operators into $L^{1}(\mu)$ are the same [DU, p. 259]. Then, a sequence $\left(f_{n}\right)$ in $L^{1}(\mu)$ lies in the range of an $L^{1}(\mu)$-valued bv-measure iff there is $g \in L^{1}(\mu)$ such that $\left|f_{n}\right| \leq g \quad \mu$-almost everywhere for any $n \in \mathbb{N}$. To prove this, it suffices to recall Grothendieck's Theorem about integral operators into $L^{1}(\mu): T: X \rightarrow L^{1}(\mu)$ is integral if and only if $T\left(B_{X}\right)$ is a lattice bounded subset of $L^{1}(\mu)$. 


\section{REFERENCES}

[AD] R. Anantharaman and J. Diestel, Sequences in the range of a vector measure, Ann. Soc. Math. Polon. Ser. I Comment. Math. Prace Mat. 30 (1991), 221-235.

[DU] J. Diestel and J. J. Uhl, Vector measures, Math. Surveys Monographs, vol. 15, Amer. Math. Soc., Providence, RI, 1977.

[P1] A. Pietsch, Quasinucleare Abbildungen in Normierten Raumen, Math. Ann. 165 (1966), 76-90.

[P2] _ Operator ideals, North-Holland, Amsterdam and New York, 1980.

[Pñ] C. Piñeiro, Operators on Banach spaces taking compact sets inside ranges of vector measures, Proc. Amer. Math. Soc. 116 (1992), 1031-1040.

[PR] C. Piñeiro and L. Rodriguez-Piazza, Banach spaces in which every compact lies inside the range of a vector measure, Proc. Amer. Math. Soc. 114 (1992), 505-517.

[Ps] G. Pisier, Factorization of linear operators and geometry of Banach spaces, CBMS Regional Conf. Ser. in Math., vol. 60, Amer. Math. Soc., Providence, RI, 1984.

[T] N. Tomczak-Jaegerman, Banach-Mazur distances and finite-dimensional operator ideals, Pitman Monographs Surveys Pure Appl. Math., vol. 38, Longman Sci. Tech., Harlow, 1989.

Departamento de análisis Matemático, Facultad de Matemáticas, Universidad de Sevilla, Aptdo. 1160, Sevilla, 41080, Spain

Current address: Departamento de Matemáticas, Escuela Politécnica Superior, Universidad de Huelva, La Rábida, Huelva, Spain

E-mail address: candido@colon. uhu.es 